\title{
Comparison Results for a Class of Variational Inequalities
}

\author{
M. R. Posteraro - R. Volpicelli
}

ABSTRACT. In this paper we study a variational inequality related to a linear differential operator of elliptic type. We give a pointwise bound for the rearrangement of the solution $u$, and an estimate for the $L^{2}$-norm of the gradient of $u$.

\section{INTRODUCTION}

Let $\Omega$ be an open bounded set of $\mathbb{R}^{n}$ and let

$$
A u=-\left(a_{i j}(x) u_{x_{j}}\right)_{x_{i}}+\left(\mathrm{b}_{i}(x) u\right)_{x_{i}}+c(x) u
$$

be a differential operator whose coefficients satisfy:

$$
\left\{\begin{array}{l}
a_{i j}, b_{i}, c \in L^{\infty}(\Omega) \\
a_{i j}(x) \xi_{i} \xi_{j} \geq|\xi|^{2}, \\
\Sigma b_{i}^{2} \leq B^{2}
\end{array} \quad \text { for a.e. } x \in \Omega, \xi \in R^{n}\right.
$$

Let $u \in H_{0}^{1}(\Omega)$ be a solution of the related variational inequality

(1.2) $a(u, U-u) \geq \int_{\Omega} f(x)(U-u) d x \quad \forall U \in H_{0}^{1}(\Omega), U, u \geq 0$

where

1991 Mathematics Subject Classification: 35J85, 35B05.

Editorial Complutense. Madrid, 1993. 


$$
a(\varphi, \psi)=\int_{\Omega}\left(a_{i j}(x) \varphi_{x_{i}} \psi_{x_{j}}-b_{i}(x) \varphi \psi_{x_{i}}+c(x) \varphi \psi\right) d x,
$$

and $f \in L^{2}(\Omega)$.

Schwarz symmetrization allows us to obtain sharp estimates for a solution $u$ of (1.2) comparing $u$ with the solution $v \in H_{0}^{1}\left(\Omega^{*}\right)$ of the «symmetrized» variational inequality

$$
\begin{aligned}
a^{\#}(v, V-v) & =\int_{\Omega^{*}} v_{x_{i}}(V-v)_{x_{i}}+B \frac{x_{i}}{|\bar{x}|} v(V-v)_{x_{i}}-c^{*}(x) v(V-v) d x \geq \\
& \geq \int_{\Omega^{*}} f^{*}(x)(V-v) d x \quad \forall \mathrm{V} \in \mathrm{H}_{0}^{1}\left(\Omega^{*}\right), \quad V, v \geq 0
\end{aligned}
$$

where $\Omega^{*}$ is the $n$-dimensional ball centered at the origin with measure $|\Omega|, f^{\prime \prime}(x), c_{-}^{*}(x)$ are the spherically symmetric decreasing rearrangements of $f(x)$ and $c_{-}(x)=\operatorname{Max}(-c(x), 0)$, respectively (see $\S 2$ ).

More precisely in this paper we show (see theorem 2.2) that the pointwise estimate

$$
u^{*}(x) \leq v(x)
$$

holds for all $x \in \Omega^{*}$. Such an estimate gives an optimal upper bound for the $L^{p}$-norm of $u$ and for the measure of the set $\{x: u(x)>0\}$ or, that is the same, an optimal lower bound for the coincidence set of $u$.

As consequence of (1.4) we derive an estimate for $a(u, u)$ and for the $L^{2}$-norm of $\nabla u$ in terms of $a^{*}(v, v)$ and the $L^{2}$-norm of $\nabla v$ respectively. Finally the previous results are applied to the obstacle problem when the obstacle is in $H_{0}^{1}(\Omega)$.

The first results in this direction, in the case $b_{i}=0, c \geq 0$, are due to Bandle-Mossino [BM] and Maderna-Salsa [MS]. In particular in [BM] the case of a non-linear operator is considered. Subsequently Alvino-Matarasso-Trombetti [AMT] considered a variational inequality of general form taking into account also the influence of the term $c_{+}(x)=\max (c(x), 0)$, but with constraints on the coefficients different from (1,1). As far as variational parabolic inequalities concern we recall [DM].

The method used was introduced by Talenti [Ta1] for an elliptic equation without lower-order terms and was extended to elliptic equations of more general form by several authors (see i.e. [AT], [ALT2], [Ba], [FP], [GT], [Ta2], [TV]). 


\section{MAIN RESULTS}

If $\Omega$ is an open bounded set of $\mathbb{R}^{n}$, we will denote by $|\Omega|$ its measure and by $\Omega^{*}$ the ball of $\mathbb{R}^{n}$ centered at the origin whose measure is $|\Omega|$. Moreover if $\varphi$ is a measurable function,

$$
\mu(t)=|\{x \in \Omega: \varphi(x)>t\}|, \quad t \in \mathbb{R},
$$

is the distribution function of $\varphi$ and

$$
\varphi^{*}(s)=\sup \{t \in \mathbb{R}: \mu(t)>s\}, \quad s \in[0,|\Omega|[,
$$

is its decreasing rearrangement. If $C_{n}$ is the measure of the n-dimensional unit ball,

$$
\varphi^{*}(x)=\varphi^{*}\left(C_{n}|x|^{n}\right), \quad x \in \Omega^{*},
$$

is the spherically symmetric decreasing rearrangement of $\varphi(x)$. For an exhaustive treatment of rearrangements we refer for example to [ALT1], [Ba], [CR], [HLP], [Ka], [Mo]. Here we just recall the well known Hardy inequality:

$$
\int_{\Omega} u(x) v(x) d x \leq \int_{0}^{|\Omega|} u^{*}(s) v^{*}(s) \mathrm{ds}
$$

where $u(x), v(x)$ are measurable functions.

The firts step to get the comparison result is to obtain a differential inequality involving the decreasing rearrangement of $u$. In the following we will consider $f_{+}, f_{-} \neq \equiv 0$. In the other cases proofs are simpler.

Theorem 2.1. Let $u$ be a solution of the variational inequality (1.2) with conditions $(1.1)$, then

$$
\begin{gathered}
-u^{* \prime}(s) \leq \frac{s^{-2+2 / n}}{n^{2} C_{n}^{2 / n}} \int_{0}^{s}\left(f^{*}(\sigma)+c_{-}^{*}(\sigma) u^{*}(\sigma)\right) d \sigma+ \\
+\frac{B s^{-1+1 / n}}{n C_{n}^{1 / n}} u^{*}(s) \text { a.e. in }[0,|u>0|] .
\end{gathered}
$$


Proof. Following [BM] we choose as test function $U$ in (1.2) $u \pm \Phi_{h}$ where

$$
\Phi_{h}(x)= \begin{cases}h & \text { if } u>t+h \\ (u(x)-t) & \text { if } t<u(x) \leq t+h \\ 0 & \text { otherwise }\end{cases}
$$

with $h>0$ and $t \in[0, \sup u[$.

We have

$$
\frac{1}{h} a\left(u, \Phi_{h}\right)=\frac{1}{h} \int_{\Omega} f \Phi_{h} d x
$$

and by ellipticity condition, letting $h$ go to 0 , we obtain

$$
-\frac{d}{d t} \int_{u>t}|\nabla u|^{2} d x \leq-\frac{d}{d t} \int_{u>t} b_{i}(x) u_{x_{i}} u d x+\int_{u>t}(f(x)-c(x) u) d x .
$$

From now on we give a sketch of the proof because the tools are standard. We have (see [ALT2])

$$
-\frac{d}{d t} \int_{u>t} b_{i}(x) u_{x_{i}} u d x \leq B t\left(-\frac{d}{d t} \int_{u>t}|\nabla u| d x\right)
$$

and by Hardy inequality

$$
\int_{u>t}(f(x)-c(x) u(x)) d x \leq \int_{0}^{\mu(t)}\left(f^{*}(\sigma)+c_{-}^{*}(\sigma) u^{*}(\sigma)\right) d \sigma .
$$

Then

$$
-\frac{d}{d t} \int_{u>t}|\nabla u|^{2} d x-B t\left(-\frac{d}{d t} \int_{u>t}|\nabla u| d x\right) \leq
$$

$$
\leq \int_{0}^{\mu(t)}\left(f^{*}(\sigma)+c_{-}^{*}(\sigma) u^{*}(\sigma)\right) d \sigma .
$$

Isoperimetric inequality [DG], Fleming-Rishel formula [FR] and Schwarz inequality give (see [ALT2], [Ta2])

$$
n C_{n}^{1 / n} \mu(t)^{1-1 / n} \leq-\frac{d}{d t} \int_{u>t}|\nabla u| \leq
$$




$$
\leq\left[-\mu^{\prime}(t)\right]^{1 / 2}\left(-\frac{d}{d t} \int_{u>t}|\nabla u|^{2}\right)^{1 / 2} .
$$

Now we observe that the right hand side of (2.2) is non negative. As a matter of fact, letting $t$ go to 0 , from (2.2) we get

$$
\int_{0}^{\mu(0)}\left(f^{*}(\sigma)+c_{-}^{*}(\sigma) u^{*}(\sigma)\right) \mathrm{d} \sigma \geq 0
$$

and then

$$
\int_{0}^{u(t)}\left(f^{*}(\sigma)+c_{-}^{*}(\sigma) u^{*}(\sigma)\right) \mathrm{d} \sigma \geq 0 \quad \forall t \in[0, \sup u]
$$

because $f^{*}(\sigma)+c_{-}^{*}(\sigma) u^{*}(\sigma)$ is a decreasing function.

Hence by (2.3) and (2.4) we have

$$
\begin{gathered}
\int_{0}^{\mu(t)}\left(f^{*}(\sigma)+c_{-}^{*}(\sigma) u^{*}(\sigma)\right) \mathrm{d} \sigma \leq \\
\leq \frac{\mu(t)^{-1+1 / n}}{n C_{n}^{1 / n}}\left[-\mu^{\prime}(t)\right]^{1 / 2}\left(-\frac{d}{d t} \int_{u>l}|\nabla u|^{2}\right)^{1 / 2} \int_{0}^{\mu(t)}\left(f^{*}(\sigma)+\right. \\
\left.+c_{-}^{*}(\sigma) u^{*}(\sigma)\right) d \sigma .
\end{gathered}
$$

Then by (2.2) and (2.3)

$$
\left(-\frac{d}{d t} \int_{u>1}|\nabla u|^{2} d x\right)^{1 / 2} \leq
$$

$$
\leq B t\left[-\mu^{\prime}(t)\right]^{1 / 2}+\left[-\mu^{\prime}(t)\right]^{1 / 2} \frac{\mu(t)^{-1+1 / n}}{n C_{n}^{1 / n}} \int_{0}^{\mu(t)}\left(f^{*}(\sigma)+c_{-}^{*}(\sigma) u^{*}(\sigma)\right) d \sigma .
$$

Using again (2.3), we obtain

$$
\begin{gathered}
\frac{n C_{n}^{1 / n} \mu(t)^{1-1 / n}}{\left[-\mu^{\prime}(t)\right]^{1 / 2}} \leq B t\left[-\mu^{\prime}(t)\right]^{1 / 2}+ \\
+\left[-\mu^{\prime}(t)\right]^{1 / 2} \frac{\mu(t)^{-1+1 / n}}{n C_{n}^{1 / n}} \int_{0}^{\mu(t)}\left(f^{*}(\sigma)+c_{-}^{*}(\sigma) u^{*}(\sigma)\right) d \sigma .
\end{gathered}
$$


Making a change of variables we get (2.1).

We consider the variational inequality (1.3) whose data are spherically symmetric. If its solution $v$ is spherically symmetric and decreasing, then repeating the above proof, it is easy to verify that all the inequalities used to get (2.1) are equalities. As in [AMT] a way to establish the existence of such a solution is given by the following proposition:

Proposition 2.1, Let us consider the differential operator

$$
A^{*} v=-\Delta v-B\left(v \frac{x_{i}}{|x|}\right)_{x_{i}}-c_{-}^{*}(x) v .
$$

Let us suppose $c_{-}^{*}=0$ or let any one of the following conditions be satisfied:

(i) there exists a non negative function $H \neq 0$ such that the Dirichlet problem

$$
\left\{\begin{array}{l}
A^{*} Z=H \quad \text { in } \Omega^{*} \\
Z \in H_{0}^{1}\left(\Omega^{*}\right)
\end{array}\right.
$$

has a non negative solution $Z$;

(ii) the first eigenvalue of the problem

$$
\left\{\begin{array}{l}
A^{*} \varphi=\lambda p(x) \varphi \quad \text { in } \Omega^{*} \\
\varphi \in H_{0}^{1}\left(\Omega^{*}\right)
\end{array}\right.
$$

where $p \in L^{n / 2}\left(\Omega^{*}\right), p(x) \not 0$ is a non negative function, is positive;

(iii) there exists $\alpha>0$ such that

$$
\begin{aligned}
& \int_{\Omega^{*}} e^{B|x|}\left(|\nabla \Phi|^{2}-B \frac{(n-1)}{|x|} \Phi^{2}-c_{-}^{*} \Phi^{2}\right) d x \geq \\
& \geq \alpha \int_{\Omega^{*}} e^{B|x|}|\nabla \Phi|^{2} d x \quad \forall \Phi \in H_{0}^{1}\left(\Omega^{*}\right) .
\end{aligned}
$$

Then (1.3) has a unique solution $v=v^{*}$. Moreover for $A^{*}$ a maximum principle holds, that is 


$$
A^{*} V \geq 0 \text { in } \Omega^{*}, \quad V \geq 0 \text { on } \partial \Omega^{*} \Rightarrow V \geq 0 \text { in } \Omega^{*} \text {. }
$$

The proof of this proposition will be given in the appendix.

The above arguments yield to state the following

Proposition 2.2. If one of the conditions of proposition 2.1 is verified then the solution $v=v^{\#}$ of (1.3) satisfies

$$
\begin{gathered}
-v^{* \prime}(s)=\frac{\mathrm{s}^{-2+2 / n}}{n^{2} C_{n}^{2 / n}} \int_{0}^{s}\left(f^{*}(\sigma)+c^{*}(\sigma) v^{*}(\sigma)\right) d \sigma+ \\
+\frac{B s^{-1+1 / n}}{n C_{n}^{1 / n}} v^{*}(s) \quad \text { a.e. in }[0,|v>0|] .
\end{gathered}
$$

At this point we are able to prove the comparison result.

Theorem 2.2. If one of the conditions of proposition 2.1 is verified and $u, v$ are solutions respectively of (1.2), (1.3) with the assumptions (l.I), then

$$
u^{*}(s) \leq v^{*}(s) \quad \text { in }[0,|\Omega|]
$$

Proof. Starting from (2.1) and (2.8) we show that, setting $w=u^{*}-v^{*}$, we will show that

(2.9) $-w^{\prime}(s) \leq B \frac{s^{-1+1 / n}}{n C_{n}^{1 / n}} w+\frac{s^{-2+2 / n}}{n^{2} C_{n}^{2 / n}} \int_{0}^{s} c_{-}^{*}(\sigma) w(\sigma) d \sigma \quad$ in $[0,|u>0|]$.

Obviously (2.9) holds for all $s \in[0, \min \{|u>0|,|v>0|\}]$, then it is trivial if $|u>0| \leq|v>0|$. We suppose $|u>0|>|v>0|$. By regularity results (see [BS]) the solution of (1.3) is in $H^{2}\left(\Omega^{*}\right)$, then $\left.\left.v^{*} \in C^{1}(] 0,|\Omega|\right]\right)$ and $v^{*^{\prime}}(|v>0|)=0$.

Hence by (2.8)

$$
\int_{0}^{|v>0|} f^{*}(\sigma) d \sigma+\int_{0}^{|v>0|} c_{-}^{*}(\sigma) v^{*}(\sigma) d \sigma=0
$$


By (2.1), taking into account that $w(s)=u^{*}(s)$ for $|v>0| \leq s \leq|u>0|$ and (2.10), we get

$$
-w^{\prime}(s) \leq B \frac{s^{-1+1 / n}}{n C_{n}^{1 / n}} w+\frac{s^{-2+2 / n}}{n^{2} C_{n}^{2 / n}} \int_{0}^{s} c_{-}^{*}(\sigma) w(\sigma) d \sigma+\frac{s^{-2+2 / n}}{n^{2} C_{n}^{2 / n}} \int_{|v>0|}^{s} f^{*}(\sigma) d \sigma
$$

for $|v>0| \leq s \leq|u>0|$.

Then we obtain (2.9) observing that by $(2.10)$

$$
\int_{0}^{|v>0|} f^{*}(\sigma) d \sigma \leq 0
$$

which implies $f^{*}(s)<0$ in $[|v>0| ;|\Omega|]$ because $f^{*}$ is a decreasing function. To get the thesis we first suppose $c_{-}^{*}>0$. Setting $V=\int_{0}^{s} c_{-}^{*}(\sigma) w(\sigma) d \sigma,(2.9)$ can be written as

$$
\left\{\begin{array}{l}
-\left(\exp \left(\frac{B s^{1 / n}}{C_{n}^{1 / n}}\right) \frac{V^{\prime}}{c_{-}^{*}}\right)^{\prime}-\frac{s^{-2+2 / n}}{n^{2} C_{n}^{2 / n}} \exp \left(\frac{B s^{1 / n}}{C_{n}^{1 / n}}\right) V \leq 0 \text { in }[0,|u>0|] \\
V(0)=0 \\
V^{\prime}(|\mathrm{u}>0|) \leq 0
\end{array}\right.
$$

We will show that $V \leq 0$. If we suppose ab absurdo $V_{+} \neq 0$, then there exists $\tilde{s}$ such that $V(\tilde{s})>0, V^{\prime}(\tilde{s})=0$ and $V_{+} \not \equiv 0$ in $[0, \tilde{s}]$. We denote by $\bar{B}$ the ball centered at the origin whose measure is $\tilde{s}$ and we consider the eigenvalue problem

$$
\left\{\begin{array}{l}
A^{*} \varphi=\lambda c_{-}^{*}(x) \varphi \quad \text { in } \tilde{B} \\
\varphi \in H_{0}^{1}(\tilde{B}) .
\end{array}\right.
$$

If we observe that

$$
A^{*} \varphi=\lambda c_{-}^{*}(x) \varphi \Leftrightarrow-\left(e^{B|x|} \varphi_{x_{i}}\right)_{x_{i}}-e^{B|x|} B \frac{(n-1)}{|x|} \varphi-e^{B|x|} c_{-}^{\#}(x) \varphi=e^{B|x|} \lambda c_{-}^{*}(x) \varphi
$$

then the first eigenvalue of $(2.12)$ can be characterized by

$$
\lambda_{1}=\min _{\Phi \in H_{\alpha}^{1}(\tilde{B})} \frac{\int_{\dot{B}} e^{B|x|}\left(|\nabla \Phi|^{2}-B \frac{(n-1)}{|\bar{x}|} \Phi^{2}-c_{-}^{*}(x) \Phi^{2}\right) d x}{\int_{\dot{B}} e^{B|x|} c_{-}^{*}(\bar{x}) \Phi^{2} d x} .
$$


Since $\tilde{B} \subset \Omega^{*}$, by (ii) of proposition 2.1 we find $\lambda_{1}>0$. On the other hand if $\varphi_{1}$ is the eigenfunction corresponding to $\lambda_{1}$, we have

$$
\begin{array}{cc}
-\varphi_{1}^{\prime}(s)-B \frac{s^{-1+1 / n}}{n C_{n}^{1 / n}} \varphi_{1}(s)-\frac{s^{-2+2 / n}}{n^{2} C_{n}^{2 / n}} \int_{0}^{s} c_{-}^{*}(\sigma) \varphi_{1}(\sigma) d \sigma= \\
=\lambda_{1} \frac{s^{-2+2 / n}}{n^{2} C_{n}^{2 / n}} \int_{0}^{s} c_{-}^{*}(\sigma) \varphi_{1}(\sigma) d \sigma \quad \text { a.e. in }[0, \bar{s}] .
\end{array}
$$

Hence setting $\Phi=\int_{0}^{s} c_{-}^{*}(\sigma) \varphi_{1}(\sigma) d \sigma$, it is easy to verify that $\lambda_{1}$ is the same as the first eigenvalue of the problem

$$
\begin{aligned}
& -\left(\exp \left(\frac{B s^{1 / n}}{C_{n}^{1 / n}}\right) \frac{\Phi^{\prime}}{c_{-}^{*}}\right)^{\prime}-\frac{s^{-2+2 / n}}{n^{2} C_{n}^{2 / n}} \exp \left(\frac{B s^{1 / n}}{C_{n}^{1 / n}}\right) \Phi= \\
& =\lambda_{1} \frac{s^{-2+2 / n}}{n^{2} C_{n}^{2 / n}} \exp \left(\frac{B s^{1 / n}}{C_{n}^{1 / n}}\right) \Phi \\
& \Phi(0)=\Phi^{\prime}(\tilde{s})=0 .
\end{aligned}
$$

By (2.13), using the variational characterization of $\lambda_{1}$ and (2.11), we get

$$
\lambda_{1} \leq \frac{\int_{0}^{s} \exp \left(\frac{B s^{1 / n}}{C_{n}^{1 / n}}\right) \frac{V_{+}^{\prime 2}}{c_{-}^{*}}-\frac{s^{-2+2 / n}}{n^{2} C_{n}^{2 / n}} \exp \left(\frac{B s^{1 / n}}{C_{n}^{1 / n}}\right) V_{+}^{2} d s}{\int_{0}^{3} \frac{s^{-2+2 / n}}{n^{2} C_{n}^{2 / n}} \exp \left(\frac{B s^{1 / n}}{C_{n}^{1 / n}}\right) V_{+}^{2} d s} \leq 0
$$

which gives the absurd.

Using again (2.11) we have

$$
\left\{\begin{array}{l}
-\left(\exp \left(\frac{B s^{1 / n}}{C_{n}^{1 / n}}\right) \frac{V^{\prime}}{c_{-}^{*}}\right)^{\prime} \leq 0 \\
V(0)=0 \\
V^{\prime}(|u>0|) \leq 0 .
\end{array}\right.
$$

Integrating between $s$ and $|u>0|$, we obtain $V^{\prime}(s) \leq 0$, that is $w(s) \leq 0$. The result in the case $c_{-}^{*} \geq 0$ can be proved by approximation techniques. 
Making use of the techniques of the proof of theorem 2.1 it is possible to estimate the $L^{2}$-norm of $\nabla u$. We have the following

Theorem 2.3. If one of the conditions of proposition 2.1 is verified and $u, v$ are solution respectively of (1.2), (1.3) with the assumptions (1.1), then

$$
\int_{\Omega}|\nabla u|^{2} d x \leq \int_{\Omega^{*}}|\nabla v|^{2} d x
$$

Proof. Starting from (2.5) and squaring both sides we get

$$
\begin{gathered}
-\frac{d}{d t} \int_{\mu>r}|\nabla u|^{2} d x \leq \\
\leq-\mu^{\prime}(t)\left[B t+\frac{\mu(t)^{-1+1 / n}}{n C_{n}^{1 / n}-} \int_{0}^{\mu(t)}\left(f^{*}(\sigma)+c_{-}^{*}(\sigma) u^{*}(\sigma)\right) d \sigma\right]^{2} .
\end{gathered}
$$

Integrating between 0 and sup $u$ and making a change of variables we obtain

$$
\begin{gathered}
\int_{\Omega}|\nabla u|^{2} d x \leq \\
\leq \int_{0}^{|u>0|}\left[B u^{*}(s)+\frac{s^{-1+1 / n}}{n C_{n}^{1 / n}} \int_{0}^{s}\left(f^{*}(\sigma)+c_{-}^{*}(\sigma) u^{*}(\sigma)\right) d \sigma\right]^{2} d s .
\end{gathered}
$$

By Theorem 2.2 we have $u^{*}(s) \leq v^{*}(s)$, which implies also $|u>0| \leq|v>0|$, therefore we conclude observing that

$$
\begin{gathered}
\int_{\Omega^{*}}|\nabla v|^{2} d x= \\
=\int_{0}^{|v>0|}\left[B v^{*}(s)+\frac{s^{-1+1 / n}}{n C_{n}^{1 / n}} \int_{0}^{s} f^{*}(\sigma)+c_{-}^{*}(\sigma) v^{*}(\sigma) d \sigma\right]^{2} d s .
\end{gathered}
$$

Using the previous results we can get also an estimate for $a(u, u)$.

Proposition 2.3. Under the same assumptions of theorem 2.2, the following estimates hold 
(a) $\quad a(u, u)+\int_{\Omega} c_{-}(x) u^{2} d x \leq a^{*}(v, v)+\int_{\Omega^{*}} c_{-}^{*}(x) v^{2} d x ;$

(B) $a(u, u) \leq a^{*}(v, v) \quad$ if $b_{i}(x)=0$.

Proof. If in (1.2) we choose as test function $U=0$ and $U=2 u$ we obtain

$$
a(u, u)=\int_{\Omega} f(x) u d x
$$

By Hardy inequality we have

$$
a(u, u)+\int_{\Omega} c_{-}(x) u^{2} d x \leq \int_{0}^{|\Omega|}\left(f^{*}(s) u^{*}+c_{-}^{*}(s) u^{*}\right) d s,
$$

and integration by parts gives

$$
\int_{0}^{|\Omega|}\left(f^{*}(s)+c_{-}^{*}(s) u^{*}\right) u^{*} d s=\int_{0}^{|\Omega|} \int_{0}^{s}\left(f^{*}(t)+c_{-}^{*}(t) u^{*}\right) d t\left(-d u^{*}(s)\right) .
$$

By (2.4) we have

$$
\int_{0}^{s}\left(f^{*}(t)+c_{-}^{*}(t) u^{*}\right) d t \geq 0,
$$

moreover (2.1), (2.8) and theorem 2.1 imply

$$
-\frac{d u^{*}}{d s} \leq-\frac{d v^{*}}{d v}
$$

Then

$$
\begin{gathered}
\int_{0}^{|\Omega|}\left(f^{*}(s) u^{*}+c_{-}^{*}(s) u^{*^{2}}\right) d s \leq \int_{0}^{|\Omega|} \int_{0}^{s}\left(f^{*}(t)+c_{-}^{*}(t) v^{*}\right) d t\left(-d v^{*}(s)\right)= \\
=\int_{0}^{|\Omega|}\left(f^{*}(s)+c_{-}^{*}(s) v^{*}\right) v^{*} d s=a^{*}(v, v)+\int_{\Omega^{*}} c_{-}^{*}(x) v^{2} d x,
\end{gathered}
$$

that is $(\alpha)$ or, which is the same, $(\beta)$ when $c_{-}(x)=0$.

If $b_{i}(x)=0$, then the bilinear form $a^{\#}(u, v)$ is symmetric and hence we get 


$$
\begin{gathered}
a(u, u)=\int_{\Omega} f(x) u d x \leq \int_{\Omega^{*}} f^{*}(x) u^{*} d x=a^{*}\left(v, u^{*}\right) \leq \\
\leq \sqrt{a^{*}(v, v)} \sqrt{a^{*}\left(u^{*}, u^{*}\right)} .
\end{gathered}
$$

Ellipticity condition, Polya Szego and Hardy inequalities give

$$
a^{\#}\left(u^{\#}, u^{\#}\right)=\int_{\Omega^{*}}\left|\nabla u^{\#}\right|^{2} d x-\int_{n^{*}} c_{-}^{*}(x) u^{\# 2} d x \leq
$$

$$
\leq \int_{\Omega}|\nabla u|^{2} d x-\int_{\Omega} c_{-}(x) u^{2} d x \leq a(u, u) .
$$

By (2.14) and (2.15) $(\beta)$ follows.

Remark. We can apply the previous results to the obstacle problem

$$
a(u, \varphi-u) \geq \int_{\Omega} f(\varphi-u) \quad \forall \varphi \in H_{0}^{1}(\Omega) \varphi, u \geq \psi
$$

where the obstacle $\psi \in H_{0}^{1}(\Omega)$ and

$$
A \psi=-\left(a_{i j}(x) \psi_{x_{i}}\right)_{x_{j}}+\left(b_{i}(x) \psi\right)_{x_{i}}+c(x) \psi \quad \text { in } L^{2}(\Omega) .
$$

Setting $\bar{u}=u-\psi$ and $g=f-A \psi$ we have that $\ddot{u}$ satisfies

$$
a(\bar{u}, \varphi-\bar{u}) \geq \int_{\Omega} g(\varphi-\bar{u}) \quad \varphi \in H_{0}^{\prime}(\Omega) \varphi, \bar{u} \geq 0 .
$$

Hence we can compare $\bar{u}$ with the solution of the symmetrized problem

$$
a^{*}(v, V-v) \geq \int_{\Omega^{*}} g^{*}(x)(V-v) d x \quad \forall V \in H_{0}^{1}\left(\Omega^{*}\right) V, v \geq 0
$$

Acknowledgement. We would like to thank prof. J. I. Díaz for having suggested to estimate the energy in complete form.

\section{APPENDIX}

Proof of Proposition 2.1. Following [AMT] at first we prove that conditions (i), (ii), (iii) are equivalent. In order to do that let us observe that 
(A.1) $\quad A^{*} Z=H \Leftrightarrow-\left(e^{B|x|} Z_{x_{i}}\right)_{x_{i}}-e^{B|x|} B \frac{(n-1)}{|x|} Z-e^{B \mid x i} c_{-}^{\#}(x) Z=e^{B|x|} H$

then the first eigenvalue of (2.6) can be characterized by the Rayleigh principle

(A.2) $\quad \lambda_{1}=\min _{\phi \in H_{0}^{t}\left(\Omega^{*}\right)} \frac{\int_{\Omega^{*}} e^{B|x|}\left(|\nabla \Phi|^{2}-B \frac{(n-1)}{|x|} \Phi^{2}-c_{-}^{*}(x) \Phi^{2}\right) d x}{\int_{\Omega^{*}} e^{B|x|} p(x) \Phi^{2} d x}$.

(i) $\Rightarrow$ (ii)

By (A.1)

$$
\lambda_{1} \int_{\Omega^{*}} e^{B|x|} p(x) \Phi_{1} Z d x=\int_{\Omega^{*}} e^{B|x|} H \Phi_{1} d x,
$$

where $\Phi_{1}$ is the first eigenfunction of (2.6). Then, since $\Phi_{1}$ has constant sign in $\Omega^{*}$, we have $\lambda_{1}>0$.

(ii) $\Rightarrow$ (iii)

Chōōsing iñ $($ iii $) \bar{p}(\bar{x}) \geq B \frac{(n-1)}{|x|}+c_{-}^{*}(x)$, and using (A.2) we get

$$
\begin{gathered}
\int_{\Omega^{*}} e^{B|x|}\left(|\nabla \Phi|^{2}-B \frac{(n-1)}{|x|} \Phi^{2}-c_{-}^{*} \Phi^{2}\right) d x-\alpha \int_{\Omega^{*}} e^{B|x|}|\nabla \Phi|^{2} d x= \\
=(1-\alpha) \int_{\Omega^{*}} e^{B|x|}|\nabla \Phi|^{2} d x-\int_{\Omega^{*}} e^{B|x|}\left(B \frac{(n-1)}{|x|} \Phi^{2}+c_{-}^{\#} \Phi^{2}\right) d x \geq \\
\geq \lambda_{1}(1-\alpha) \int_{\Omega^{*}} e^{B|x|} p(x) \Phi^{2}-\alpha \int_{\Omega^{*}} e^{B|x|}\left(B \frac{(n-1)}{|x|} \Phi^{2}+c_{-}^{\#} \Phi^{2}\right) d x \geq \\
\geq\left(\lambda_{1}-\alpha \lambda_{1}-\alpha\right) \int_{\Omega^{*}} e^{B|x|}\left(B \frac{(n-1)}{|x|} \Phi^{2}+c_{-}^{*} \Phi^{2}\right) d x .
\end{gathered}
$$

Taking $0<\alpha<\frac{\lambda_{1}}{\lambda_{1}+1}$, (iii) follows.

(iii) $\Rightarrow$ (i) 
By (iii) the problem

$$
\left\{\begin{array}{l}
-\left(e^{B|x|} Z_{x_{i}}\right)_{x_{i}}-e^{B|x|} B \frac{(n-1)}{|x|} Z-e^{B|x|} c_{-}^{\#}(x) Z=e^{B|x|} H \quad \text { in } \Omega^{*} \\
Z \in H_{0}^{1}\left(\Omega^{*}\right)
\end{array}\right.
$$

has a unique solution. Moreover this solution is positive. As a matter of fact setting $Z_{-}=\max (-Z, 0)$ by (A.3)

$$
\begin{aligned}
& \alpha \int_{{ }_{12}} e^{B|x|}|\nabla Z|^{2} d x \leq \int_{\pi^{\circ}} e^{B|x|}\left(\left|\nabla Z_{-}\right|^{2}-B \frac{(n-1)}{|x|} Z_{-}^{2}-c_{-}^{\#} Z_{-}^{2}\right) d x= \\
& =-\int_{\Omega^{\prime}} e^{B|x|} H Z_{-} d x \leq 0
\end{aligned}
$$

and then $Z_{-}=0$.

With similar arguments, by (iii), we obtain (2.7).

Now we observe that $(1.3)$ is equivalent to the variational inequality

$$
\begin{gathered}
\int_{\Omega^{*}} e^{B|x|}\left[v_{x_{i}}(\Phi-v)_{x_{i}}-B \frac{(n-1)}{|x|} v(\Phi-v)-c_{-}^{\#}(x) v(\Phi-v)\right] d x \geq \\
\geq \int_{\Pi^{*}} e^{B|x|} f^{*}(x)(\Phi-v) d x \quad \forall \Phi \in H_{0}^{\prime}\left(\Omega^{*}\right) \Phi, v \geq 0
\end{gathered}
$$

which, by (iii), has a unique solution $v$.

To prove that $v$ is decreasing for the sake of simplicity we suppose $v$ sufficiently smooth. Setting $r=|x|, R=\left(\frac{|v>0|}{C_{n}}\right)^{1 / n}, V=v_{r}$ and deriving with respect to $r$ the equation

$$
A^{*} v=f^{*} \quad \text { in }\{x: v(x)>0\}
$$

we obtain

$$
\begin{gathered}
-V_{r}-\frac{(n-1)}{r}-V_{r}-B V_{+}+\left(\frac{(n-1)}{r^{2}-}-B \frac{(n-1)}{r}-c_{-}^{\#}(x)\right) V= \\
=f_{r}^{*}-B \frac{(n-1)}{r^{2}} v+\left(c_{-}^{*}\right)_{r} v \quad \text { in }[0, R] .
\end{gathered}
$$

Since $f_{r}^{*}-B \frac{(n-1)}{r^{2}} v \leq 0$, we have 


$$
A^{*} V+\frac{(n-1)}{|x|^{2}} V \leq 0
$$

and $V(R) \leq 0$.

We conclude observing that the operator $A^{\prime \prime}+\frac{(n-1)}{|x|^{2}}$ has the property (2.7).

\section{References}

[ALT1] Alvino, A.-Lions, P. L.-TrombetTI, G.: On optimization problems with prescribed rearrangements, Nonlinear Analysis, T.M.A., 13 (1989), 185-220.

[ALT2] Alvino, A.-Lions, P. L.-TrombetTI, G.: Comparison results for elliptic and parabolic equations via Schwarz symmetrization, Ann. Inst. Henri Poincarè, (2) 7 (1990), 37-65.

[AMT] Alvino, A.-MAtarasso, S.-Trombett,, G.: Variational Inequalities and Rearrangements, Rend. Acc. Naz. Linc., (1992).

[AT] Alvino, A.-Trombett, G.: Sulle migliori costanii di maggiorazione per una classe di equazioni ellitiche degeneri, Ricerche Mat., 27 (1978), 413-428.

[Ba] BandLE, C.: Isoperimetric Inequalities and Applications, Monographs and Studies in Math., No. 7, Pitrnan, London, 1980.

[BM] BANDLE, C.-Mossino, J.: Rearrangement in Variational Inequalities, Ann. Mat. Pura e Appl, 138 (1984), 1-14.

[BS] Brezis, H. R.-STAMPACCHIA, G.: Sur la régularité de la solution d'inéquations elliptiques, Bull. Soc. Math. France, 96 (1968), 153-180.

[CR] CHONG, K. M.-RICE, N. M.: Equimeasurable rearrangements of functions, Queen's papers in pure and applied mathematics, No. 28, Queen's University, Ontario, 1971.

[DG] DE GIORGI, E.: Su una teoria generale della misura $(\mathrm{r}-1)$-dimensionale in uno spazio ad $r$ dimensioni, Ann. Mat. Pura e Appl., 36 (1954), 191213.

[DM] DiAz, J. I.-Mossino, J.: Isoperimetric inequalities in the parabolic obstacle problem, to appear in Joumal de Math. Pures et App.

[FP] FERONE, V.-POSTERARO, M. R.: Symmetrization results for elliptic equations with lower order terms, Atti Sem. Mat. Fis. Modena, 39 (1991), 47-61.

[FR] FLEMING, W.-RISHEL, R.; An integral formula for total gradient variation, Arch. Math., 11 (1960), 218-222.

[GT] Giarrusso, E.-TrombetT, G.: Estimates for solutions of elliptic equations in a limit case, Bull. Austral. Math. Soc., 36 (1987), 425-434.

[HLP] Hardy, G. H.-LitTlewood, J, E.-PolyA, G.: Inequalities, Cambridge University Press, 1964. 
[Ka] KAWHOL, B.: Rearrangements and convexiry of level sets in PDE, Lecture Notes in Math., No. 1150, Springer, Berlin-New York, 1985.

[MS] Maderna, C.-SAlsa, S.: Some Special Properties of Solutions to Obstacle Problems, Rend. Sem. Mat. Univ. Padova, 71 (1984), 121-129.

[Mo] Mossino, J.: Inégalités isopérimétriques et applications en physique, Collection Travaux en Cours, Hermman, Paris, 1984.

[Tal] TALENT1, G.: Elliptic equations and reattangements, Ann. Scuola Noim. Sup. Pisa, (4) 3 (1976), 697-718.

[Ta2] TALENTI, G.: Linear Elliptic P.D.E.'s: Level Sets, Rearrangements and a priori Estimates of Solutions, Boll. U.M.I., (6) 4-B (1985), 917-949.

[TV] TROMBETTI, G.-VÁZQUEZ, J. L.: A symmetrization result for elliptic equations with lower order terms, Ann. Fac. Sci. de Toulose, 7 (1985), $137-150$

Dip. di Matematica ed Appl.

QRenato Caccioppolis

Universitá di Nappoli

Complesso Monte S. Angelo

80126 Napoli

Italy

Recibido: 1 de diciembre de 1992 